\title{
Acquired hemophilia A
}

\begin{abstract}
Acquired hemophilia A (AHA) is a rare but potentially life threatening bleeding disorder caused by the development of auto antibodies against circulating factor VIII. Age distribution is bimodal with a first peak occurring among young women in postpartum period and a second major peak among elderly patients. Fifty percent of cases are idiopathic whereas the remaining 50\% are associated with pregnancy, autoimmune conditions, malignancies and drugs. Unlike severe congenital hemophilia in which joint bleeds are more common, bleeding into skin (purpura) and soft tissue bleeds are more common in AHA. Diagnosis of acquired AHA should be considered in an elderly patient who presents with bleeding and an increased aPTT. Early diagnosis and prompt treatment are necessary in this life-threatening condition especially with a very high inhibitor titer for the better outcome of the patient. We report a case of an elderly male who presented with a very high titer of factor VIII inhibitors and was successfully treated.
\end{abstract}

Keywords: hemorrhagic disorder, postpartum, hemarthroses, desmopressin, azathioprine
Volume 4 Issue 6 - 2017

\section{Javed MU, Fatima M, Malik NA, Imran A, Zafar A}

Department of Hematology, Chughtais Lahore Lab, Pakistan

Correspondence: Usman Javed M, Department of Hematology, Chughtais Lahore Lab, 10 $0^{\text {th }}$ Jail Road Main Gulberg Lahore, Pakistan, Tel +92300646I569, Email dr_usman_javed@yahoo.com

Received: March 24, 2017| Published: July 10, 2017
Abbreviations: AHA, acquired hemophilia A; APTT, activated partial thromboplastin time; CPA, cyclophosphamide; aPCC, activated prothrombin complex concentrate; LAC, lupus anticoagulant; FEIBA, factor eight inhibitor bypass activity; rFVIIa, activated recombinant factor VII

\section{Introduction}

Acquired hemophilia $\mathrm{A}$ is a rare but often life-threatening hemorrhagic disorder characterized by an auto antibody directed against coagulation factor VIII. Fifty per cent of cases are idiopathic whereas the remaining $50 \%$ are associated with pregnancy, autoimmune conditions, malignancies and drugs. ${ }^{1}$ Acquired hemophilia is significantly rarer than the inherited form, affecting around 2 per million of the population. ${ }^{2}$ Its distribution is bimodal, with the first peak occurring among young women in the postpartum period and the second among elderly patients, in whom it is frequently associated with autoimmune disease, malignant disease and allergic drug reactions. ${ }^{3}$ In contrast with congenital hemophilia, which is commonly characterized by hemarthroses, hemorrhages in patients with acquired hemophilia involve most frequently soft tissues. ${ }^{4}$ The first step in diagnosis of AHA is tracking signs of bleeding tendency, particularly in the elderly, in the clinical setting and testing for prolongation of activated partial thromboplastin time (APTT) in the laboratory. The next step is to review patient's medical history by considering the impact of any underlying conditions associated with AHA. ${ }^{5}$ A prolonged aPTT may be attributable to coagulation factor deficiencies, lupus anticoagulant or heparin therapy. So mixing tests are performed to distinguish between factor deficiency and the presence of any inhibitor. FVIII inhibitors are time and temperaturedependent, therefore mixing studies performed immediately and after $2 \mathrm{~h}$ of incubation should be compared. Prolongation of the aPTT in a mixture of patient and normal plasma after a 1-2 hours incubation compared to an immediate mix is typical of FVIII auto antibodies. In addition, factor assays should be repeated at higher serial dilutions of the test plasma, which will attenuate the effect of the inhibitor or lupus anticoagulant on the factor measurement. ${ }^{6}$
The inhibitor can be roughly quantified using the Bethesda assay, which measures residual FVIII after incubation of patient plasma with normal plasma for $2 \mathrm{~h}$ at $37^{\circ} \mathrm{C}$ : I Bethesda Unit $(\mathrm{BU}) / \mathrm{ml}$ is the quantity of inhibitor that neutralizes $50 \%$ of clotting factor activity in normal plasma. ${ }^{7}$ To treat bleeds recombinant factor VIIa and activated prothrombin complex concentrate are equally efficacious but both are superior to factor VIII or desmopressin. Immunosuppression should be started as soon as the diagnosis is made. Commonly used regimens are steroids alone or combined with cytotoxic agents like azathioprine, and cyclosporine and anti-neoplastic agents such as cyclophosphamide (CPA), mercaptopurine, and vincristine. Rituximab is being used more widely but current evidence does not suggest that it improves outcomes or reduces side effects. ${ }^{6,8}$

\section{Case presentation}

A 60 years old male presented with extensive purpura and pain in left thigh and gluteal region. Patient had a history of diabetes and hypertension for the past 3years for which he was taking oral hypoglycemics (glimepiride, glucophage) and anti-hypertensives (amlodipine). Over the past 6months, he had multiple episodes of purpura including right arm, right thigh, gluteal region and pain in right knee joint. Meanwhile he also suffered from Tinea cruris. He was prescribed pain killers (paracetamol, tramadol), anti-fungals (fluconazole, topical hydrocortisone/miconazole) and antibiotics (ciprofloxacin). Two months before presenting to the Hemophilia society he had an episode of hemetemesis for which he was given Vitamin $\mathrm{K}$ and tranexamic acid. He was admitted in emergency and laboratory tests revealed prolonged aPTT, normal platelet count and anemia $(\mathrm{Hb} 5.6 \mathrm{~g} / \mathrm{dl})$. He was transfused 2 units of packed red cells.

He was finally referred to our hemophilia society with purpura of left thigh. Coagulation profile was done and showed prolonged aPTT (58sec, Normal 24-32sec), normal PT/INR and normal bleeding time (3min, Normal $<7 \mathrm{~min}$ ). Hb was $13.9 \mathrm{~g} / \mathrm{dl}$, TLC was $10.5 \mathrm{x} 10^{\wedge} / 1$ and platelet count was $258 \times 10^{\wedge} 9 / 1$. Hepatitis B and C serology were negative and PSA and CEA levels were within normal limits. Mixing studies showed no correction of aPTT. Factor assays showed factor 
VIII level of $0.2 \%$ and factor IX level of $79 \%$. Factor VIII inhibitor levels were quantified by using Bethesda assay and was found to be very high i.e $5800 \mathrm{BU} / \mathrm{ml}$. He was treated with activated prothrombin complex concentrate (aPCC); brand name FEIBA i.e factor eight inhibitor bypassing agent (50units/kg) and responded well to the therapy. Immunosuppressive therapy with prednisone $(1 \mathrm{mg} / \mathrm{kg} /$ day $)$ and azathioprine ( $3 \mathrm{mg} / \mathrm{kg} /$ day in divided doses) was started. IVIG was not given to the patient. Two weeks later, he came again with purpura of extensor muscles of the back and was given FEIBA. Bethesda assay was repeated and was found to be $3600 \mathrm{BU} / \mathrm{ml}$. Steroid therapy was tapered off slowly over a period of 6 weeks but azathioprine was continued. During a follow up of 5 months, patient had no further episode of hemorrhage.

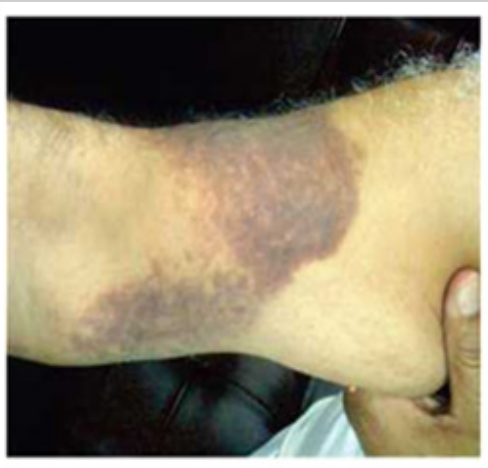

(A)

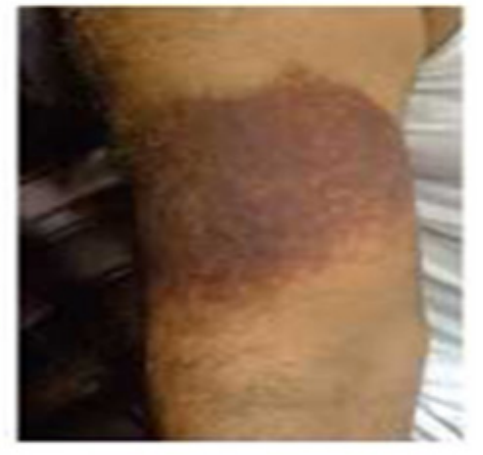

(B)

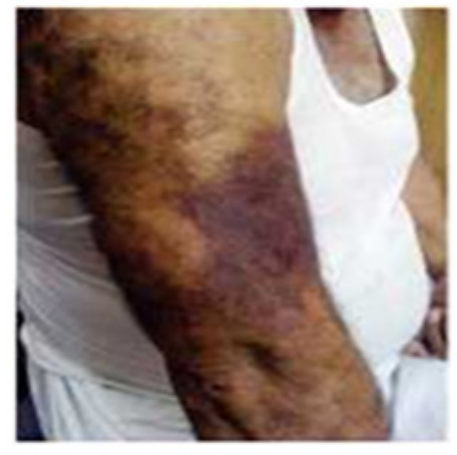

(C)

Figure I Multiple episodes of purpura
A. At right arm
B. At right thigh and
C. Gluteal region and pain in right knee joint

\section{Discussion}

AHA is a rare but life threatening bleeding disorder that typically occurs in old age in whom it is frequently associated with autoimmune diseases, malignant diseases and allergic drug reactions. According to recent data the incidence in the subjects aged $<65$ years is 0.26 per million per year but increases up to 5.97 in those aged 65-85 years. These data are likely to be underestimated because of undiagnosed and unreported cases. ${ }^{1,2}$ If isolated aPTT is prolonged in elderly patients who present with bleeding, the diagnosis is AHA should be considered. The mixing test reveals presence of inhibitor of coagulation. A prolonged aPTT can be due to coagulation factor deficiencies, lupus anticoagulant (LAC) or heparin therapy. Prolonged aPTT is observed in presence of LAC which is not corrected by normal plasma, but in this case no bleeding tendency is shown. When patient's plasma is diluted with normal plasma it leads to dilution of autoantibody and normalizes the activity of factors other than FVIII which remains inhibited even by the diluted specific autoantibody. ${ }^{6}$ The diagnosis is then confirmed and inhibitor is quantified by a Bethesda positive assay. 3,7

When AHA is diagnosed, the possible underlying disease responsible for immunologic complication should be investigated which in most cases in elderly patients is secondary to hematological or solid malignancy or drugs (antibiotics, phenytoin, methyldopa, interferon, fludarabine and clopidogrel). However, about half of the cases are apparently idiopathic. ${ }^{1,3}$ Our case report confirms the complexity of management of AHA in whom the clinical picture is complicated by comorbidities, intake of drugs and high antibody titer. The presence of comorbidities like diabetes mellitus, hypertension, high body mass index, antithrombotic treatment is a challenging issue in the management; as hypertension is a major risk factor for intracranial bleeding in such patients and diabetes along with hypertension may increase the risk of ischemic heart disease., ${ }^{910}$ The bleeding episodes lead to with drawl of such treatments and use of bypassing agents (APCCs, rFVII) with increased risk of thromboembolism. This risk is increased by immobilization, muscle bleeding or by coagulation cascade activation induced during sepsis. An accurate balance of bleeding and thrombotic risk should be taken care of while treating bleeding complications in terms of doses and duration of bypassing agent administration. At present, bypassing agents, such as factor eight inhibitor bypass activity (FEIBA) and activated recombinant factor VII (rFVIIa) are the only coagulation factor concentrates available for the treatment of bleeds in inhibitor patients. ${ }^{11}$ Evidence of bypassing agent thrombogenicity is limited therefore; they should be given in life threatening bleed. Patients with high antibody titer or high level of Bethesda assay need more aggressive and long term therapy. ${ }^{6,8}$ Appropriate therapeutic approach is crucial for favorable outcome as bleeding episodes in AHA prove to be life threatening if left untreated. It is associated with a high rate of morbidity and mortality as severe bleeds occur in $90 \%$ of patients and mortality is high ranging from $8-22 \%$. Therefore, once the patient has been diagnosed, he should be treated immediately for bleeding episodes and antibody eradication. ${ }^{4,5,6}$

\section{Acknowledgements}

None.

\section{Conflict of interest}

The author declares no conflict of interest.

\section{References}

1. Franchini M, Lippi G. Acquired hemophilia A. Adv Clin Chem. 2011;54:71-80. 
2. Collins PW, Hirsch S, Baglin TP, et al. Acquired haemophilia A in the United Kingdom: a 2-year national surveillance study by the United Kingdom Haemophilia Centre Doctors' Organistaion. Blood. 2007;109(5):1870-1877.

3. Woods S, Varghese B. Acquired hemophilia A presenting in an elderly man. CMAJ. 2007;177(4):341-342.

4. Franchini M, Lippi G. Acquired factor VIII inhibitors. Blood. 2008;112(2):250-255.

5. Sakurai Y, Takeda T. Acquired hemophilia a: a frequently overlooked autoimmune hemorrhagic disorder. J Immunol Res. 2014;2014:320674.

6. Huth-Kühne A, Baudo F, Collins P, et al. International Recommendations On The Diagnosis And Treatment Of Patients With Acquired Hemophilia A. Haematologica . 2009;94(4):566-575.
7. Delgado J, Jimenez-Yuste V, et al. Acquired haemophilia: review and meta-analysis focused on therapy and prognostic factors. Br J Haematol. 2003;121(1):21-35.

8. Collins PW. Management of acquired haemophilia A. J Thromb Haemost. 2011;9(Suppl 1):226-235.

9. Von Drygalski A, Kolaitis NA, Bettencourt R, et al. Prevalence and risk factors for hypertension in hemophilia. Hypertension. 2013;62(1):209215.

10. Kulkarni R, Soucie JM, Evatt BL. Prevalence and risk factors for heart disease among males with hemophilia. Am J Hematol. 2005;79(1):36-42.

11. Tjønnfjord GE, Holme PA. Factor eight inhibitor bypass activity (FEIBA) in the management of bleeds in hemophilia patients with hightiter inhibitors. Vasc Health Risk Manag. 2007;3(4):527-531. 